\title{
V. - TENEUR EN VITAMINE B12 DU FOIE ET DU CONTENU DE L'INTESTIN
}

\author{
PAR \\ R. FEVRIER, J. P. VACHEL, M. MICHEL \\ Station de Recherches sur L'Elevage, Jouy-en-Josas
}

De nombreux auteurs ont montré qu'il existait des relations encore mal connues entre l'action des antibiotiques et le métabolisme de la vitamine $\mathrm{BI}$.

Pour obtenir des informations plus complètes sur ce point, nous avons effectué des dosages de vitamine BI2 sur les échantillons des contenus du tube digestif prélevés pour les études précédemment relatées (IV), ainsi que sur le foie des mêm es animaux.

\section{Méthode de dosage}

I a méthode microbiologique employée dérive de celle décrite par BURKHOLDER (P. R.) et al. (I95I) ; elle utilise comme organisme-test un mutant d'Escherichia Coli souche Ir3-3.

Dans des conditions standardisées, la croissance du germe est proportionnelle à la concentration en vitamine BI2. Nous avons vérifié que la méthionine, qui peut aussi stimuler la croissance du germe, n'interférait pas.

\section{I $^{0}$ Préparation des milieux}

BOUILLON DE CULTURE (pour l'inoculum).
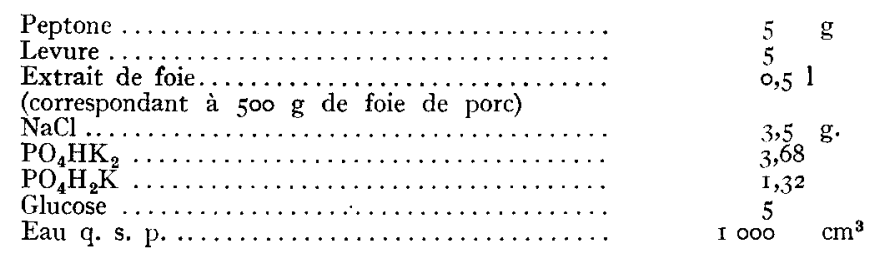

Atutoclavage I2 minutes à II $2^{\circ} \mathrm{C}$.

Ce milieu est distribué avant autoclavage en tubes de $x \mathrm{~cm}^{3}$. 
GÉLOSE NUTRITIVE.

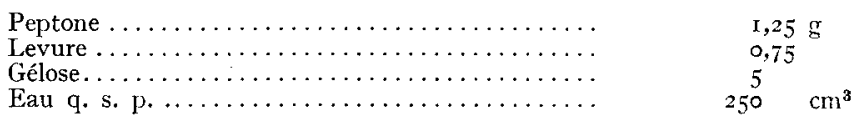

BOUILLON POUR IE DOSAGE. Acides aminés:

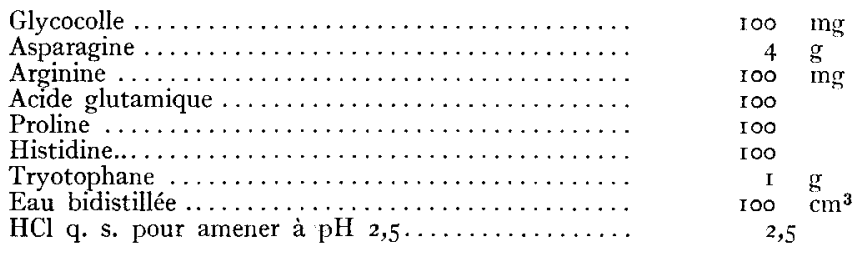

Cette solution est autoclavée I 2 minutes à II $2^{\circ} \mathrm{C}$.

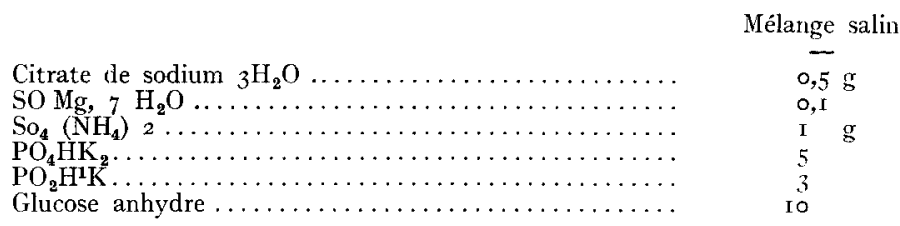

Ces produits sont broyés et mélangés à sec. Le bouillon nécessaire au dosage de la semaine est préparé comme suit :

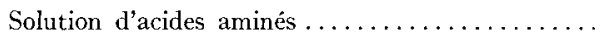

Mélange salin $\ldots \ldots \ldots \ldots \ldots \ldots \ldots \ldots \ldots \ldots \ldots \ldots \ldots \ldots \ldots \ldots \ldots \ldots \ldots \ldots \ldots \ldots$
Eau bidistillée $:$ q. s. p. $\ldots \ldots \ldots \ldots \ldots \ldots$

Le $\mathrm{pH}$ obtenu est de 6,5 - autoclavage $\mathrm{I} 2$ minutes à II $2^{\circ} \mathrm{C}$.

\section{$2^{\circ}$ Culture du germe}

La souche d'E. coli est repiquée tous les I5 jours sur gélose inclinée.

Pour préparer l'inoculum, on ensemence, la veille du dosage, un tube de $\mathrm{Io}^{\mathrm{c}} \mathrm{cm}^{3}$ de bouillon à l'anse de platine, à partir de la culture sur gélose. I'incubation dure I6 heures à $37^{\circ} \mathrm{C}$.

Le bouillon nécessaire au dosage $-5 \mathrm{~cm}^{3}$ par tube - est ensemencé avec I,25 p. roo de cette culture.

\section{$3^{0}$ Mise en solution de la vitamine B12}

Io $\mathrm{g}$ d'échantillon à doser sont broyés au mixer avec $190 \mathrm{~cm}^{3}$ d'eau bidistillée.

On prélève ro $\mathrm{cm}^{3}$ de la suspension, que l'on met dans une fiole de $100 \mathrm{~cm}^{3}$ avec $20 \mathrm{~cm}^{3}$ de solution de $\mathrm{CNK}$ à $50 \mathrm{mcg} / \mathrm{cm}^{3}$. Après autoclavage (Io minutes à $\mathrm{II} 2^{\circ} \mathrm{C}$ ) et refroidissement, on ajoute $20 \mathrm{~cm}^{3} \mathrm{de}$ tampon acide acétique, acétate de sodium à $\mathrm{pH} 4,5$, pour floculer les protéines. La solution est transférée dans une fiole jaugée de $100 \mathrm{~cm}^{3}$. On rince et complète à roo $\mathrm{cm}^{3}$ avec de l'eau bidistillée. Après filtration sur 
filtre à plis, la solution est ajustée à $\mathrm{pH}$ 6,5 et diluée pour amener le titre en vitamine $\mathrm{Br} 2$ à I millimicrogramme, d'après le titre prévu.

\section{$4^{0}$ Mesure turbidimétrique à l'électrophotomètre}

Chaque solution est distribuée dans des tubes, en triple exemplaire, à raison de $0, \mathrm{x}-0,4-0,6$ et $0,8 \mathrm{~cm}^{3}$.

I a gamme étalon est faite à partir d'une solution de BI2 pure à la concentration de I millimeg/ $/ \mathrm{cm}^{3}$.

On ajoute ensuite $5 \mathrm{~cm}^{3}$ de bouillon ensemencé par tube.

Les tubes sont placés au bain-marie à $37^{\circ}$ pendant $4 \mathrm{~h} 30$.

Après ce délai, la croissance est stoppée à l'aide de 3 gouttes de formol par tube. Puis on fait la lecture de la turbidité au photomètre.

$I_{1}$ a moyenne des chiffres obtenus pour chaque concentration étalon (pourcentage de transmission) est portée en ordonnée sur du papier millimétrique; on place en abscisses les concentrations de $\mathrm{BI} 2$ et on trace la droite représentative.

Les concentrations en $\mathrm{B} 12$ des solutions à doser sont déterminées sur la courbe, d'après leurs mesures de turbidité, et multipliées par l'inverse de la dilution.

\section{$5^{\circ}$ Résultat des dosages}

Ils sont indiqués dans le tableau suivant (échantillons bruts) :
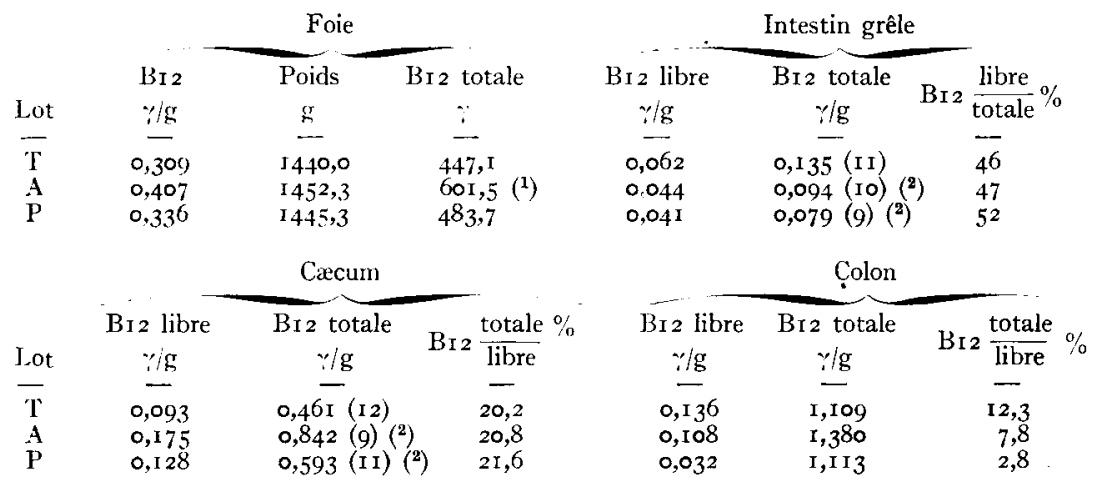

On constate les faits suivants :

I. - Foie: $\mathrm{Il}$ y a une augmentation du potentiel vitaminique $\mathrm{BI} 2$ du foie total, augmentation importante et significative dans le lot auréomycine seulement. Celle-ci est due uniquement à l'augmentation de la concentration de vitamine $\mathrm{BI} 2 \mathrm{du}$ foie frais, le poids total du foie étant sensiblement le même dans les trois lots.

2. - Si l'on se rappelle que le lot auréomycine est le seul à recevoir de la vitamine $\mathrm{BI} 2$ en plus d'un antibiotique, il semble que les anti- 
biotiques n'augmentent la concentration vitaminique du foie que dans le cas où la ration contient de la BI2.

Ces résultats paraissent être en accord avec ceux de CALET et al. obtenus chez le rat. Ces auteurs montrent qu'un régime renfermant très peu de vitamine BI2 permet un abondant stockage hépatique s'il contient de l'auréomycine et qu'au contraire, l'auréomycine n'a aucune influence sur les réserves du foie en BI2 quand la ration est totalement dépourvue de cette vitamine.

3. - I1 se produit dans le tractus intestinal de tous les animaux, quel que soit leur traitement, une synthèse considérable de vitamine B12. Pour un animal en fin d'engraissement consommant par jour environ $3 \mathrm{~kg}$ de matière sèche avec auréomycine, l'ingestion quotidienne de BI2 est au minimum de $\mathrm{I} 6 \mathrm{mcg} / \mathrm{kg} \times 3 \mathrm{~kg}$ soit $48 \mathrm{mcg}$. Pour un animal des lots témoin et pénicilline, elle est pratiquement nulle. En revanche, 1'animal excrétant environ 2 ooo $\mathrm{g}$ de fèces bruts par jour exporte : 2200 à 2800 mcg de vitamine BI2 par jour.

4. - L'importance de cette synthèse intestinale nous permet de considérer comme tout à fait négligeable la présence du facteur $\mathrm{Br} 2$ dans les ingesta d'un seul des trois lots, puisqu'il se trouve ultérieurement en quantités importantes dans le tractus intestinal de tous les animaux. On peut ainsi attribuer d̀ l'auréomycine seule, et non pas à l'auréomycine + vitamine B12, les différences significatives de croissance ou de stockage vitaminique dans le foie observées dans le lot recevant de l'auréomycine et de la vitamine $\mathrm{Br} 2$.

Nous devons cependant noter que de nombreux travaux ont mis en évidence l'influence sur la croissance de vitamine BI2 ajoutée à la ration.

5. - Dans l'intestin grêle, les teneurs en BI2 des lots recevant des antibiotiques sont significativement inférieures à celles du lot témoin ; dans le caecum, ces teneurs sont au contraire significativement supérieures à celles du lot témoin.

\section{REFÉRENCES BIBLIOGRAPHIQUES}

Burkholder (P. R.). - - "Determination of vitamin BI2 with a mutant strain of Escherichia Coli ». Science, $114 ; 459$, I95I.

Calet (C.), Rerat (A.), JacQuot (R.). - "Action d'épargne des antibiotiques pour quelques vitamines du groupe B ». C. R. Acad. Sci. $236 ; 2340-42$, I953.

Caiet (C.), Rerat (A.), Jacquot (R.). - "Signification des relations entre auréomycine et vitamine BI2 ». C.R. Acad. Sci., I954. 